\title{
Photolithographic micromolding of ceramics using plasma etched polyimide patterns
}

\author{
J. A. Bride, S. Baskaran, N. Taylor, and J. W. Halloran \\ Department of Materials Science and Engineering, University of Michigan, Ann Arbor, \\ Michigan 48109-2136 \\ W. H. Juan and S. W. Pang \\ Solid State Electronics Laboratory, Department of Electrical Engineering and Computer Science, \\ University of Michigan, Ann Arbor, Michigan 48109-2136 \\ M. O'Donnell \\ Biomedical Ultrasonics Laboratory, Department of Electrical Engineering and Computer Science, \\ University of Michigan, Ann Arbor, Michigan 48109-2136
}

(Received 19 July 1993; accepted for publication 2 October 1993)

Features as fine as $4 \mu \mathrm{m}$ with high aspect ratio were produced from ceria-zirconia ceramic using a thick plasma-etched polyimide layer as a micromold.

Photolithography is a highly advanced method for fabricating features in the micron and submicron size range. Scmiconductor proccssing methods have been applicd to fabricate microsensors and microactuators with high throughput and excellent dimensional control of small features, usually from silicon. It is clearly desirable to extend these methods to microfabricate other materials, such as piezoelectric ceramics, where such fine scale high aspect ratio features are required for high resolution ultrasonic imaging. ${ }^{1}$ We have explored the extensions of these methods to ceramic materials using, a photolithographically fabricated pattern as a "micromold" for forming small features in a ceramic. We find it possible to replicate deep features from the polyimide onto a conventional ceramic green tape made from submicron powder. The technique is applicable to any powder-processed ceramic.

Fine features with a high aspect ratio are a particular goal of this work. Fabrication of such features in polymer has been demonstrated by using the lithographic galvanoformung abformung (LIGA) process ${ }^{2}$ and reactive ion etching (RIE). ${ }^{3}$ Although LIGA can form high aspect ratio structures in polymer, it requires a high intensity synchrotron $x$-ray source which is costly and not readily available. It is also difficult to generate high aspect ratio structures with conventional RIE, because of the slow etch rate and low selectivity. In this work a multipolar electron cyclotron resonance (ECR) source is used to generate a high density plasma with low ion energy. ${ }^{4}$ This plasma system provides a fast etch rate, residue-free surface, and anisotropic etch profile for fabrication of high aspect ratio structures.

We pattern ceramic by impressing a plasma-etched polyimide pattern onto a soft ceramic "green tape." We made the green tape from a high quality submicrometer powder of a ceria-zirconia alloy, chosen as a convenient model system.

The ceramic green tape was a flexible film, $280 \mu \mathrm{m}$ thick, consisting of a 55 vol \% submicron ceramic powder in 45 vol \% plasticized ethylmethacrylate (EMA). The ceramic was a Tosoh TZ-12CE, a $12 \mathrm{~mol} \% \mathrm{CeO}_{2}-88$ mol \% $\mathrm{ZrO}_{2}$ powder with a specific surface area of $9.4 \mathrm{~m}^{2}$ $\mathrm{g}$ and an average particle size of $0.3 \mu \mathrm{m}$. A conventional tape casting slip was prepared by dispersing this powder in a 60 wt \% ethanol-40 wt \% methylcthylketonc (MEK) solution at a solid loading of 30.2 vol \%, using a Witco Emphos PS-21A phosphate ester dispersant at a level of $0.44 \mathrm{wt} \%$ of the dry ceramic. To 100 parts by weight of this dispersion, we added 25.5 parts of an Rohm and Haas Acryloid B-7 EMA binder solution ( $\mathrm{MW}_{w}=124000 \mathrm{Dal}-$ tons by gel permeation chromatography), 3.56 parts of butyl benzyl phthalate plasticizer, and 3.56 parts of 400 Dalton polyethylene glycol. The complete slip was ball milled about $4 \mathrm{~h}$ to homogenize it prior to tape casting. This slip was doctor-bladed onto a mylar substrate using a ceramic tape casting machine, and dried at room temperature.

High aspect ratio structures were generated in polyimide by etching in an $\mathrm{O}_{2}$ plasma with $\mathrm{Ti}$ as an etch mask using a trilayer resist scheme. The trilayer resist consists of DuPont Pyralin PI-2611 polyimide, Ti, and Shipley AZ5214 photoresist. The polyimide film was coated on 100 -mm-diam wafers with $\pm 5 \%$ uniformity across the wafer. A $0.2-\mu \mathrm{m}$-thick Ti layer was evaporated on top of the polyimide as a mask for the subsequent $\mathrm{O}_{2}$ plasma etching of polyimide. To define patterns on the Ti mask, a $1.4-\mu \mathrm{m}-$ thick AZ5214 photoresist was spun on the Ti mask and patterned using conventional optical lithography. The $\mathrm{Ti}$ layer was etched by $\mathrm{RIE}$ in an $\mathrm{Ar} / \mathrm{BCl}_{3}$ plasma with the developed photoresist as an etch mask. In an $\mathrm{O}_{2}$ plasma, $\mathrm{Ti}$ is oxidized to form $\mathrm{TiO}_{x}$, serving as a durable mask since $\mathrm{TiO}_{x}$ has a very low sputtering yield. Polyimide was etched in the high density and low perssure $\mathrm{O}_{2}$ plasma generated by a multipolar ECR source excited by a $2.45 \mathrm{GHz}$ microwave power supply. The wafer was placed on top of a 13.56 $\mathrm{MHz}$ rf powered stage with the temperature of the stage controlled at $-30^{\circ} \mathrm{C}$. A typical etch condition is $750 \mathrm{~W}$ microwave power and $300 \mathrm{~W}$ rf power at $0.5 \mathrm{mT}$ Torr and 20 sccm $\mathrm{O}_{2}$. The polyimide etch rate is $0.9 \mu \mathrm{m} / \mathrm{min}$ with an etch selectivity of better than 1000:1 between polyimide and the Ti mask. Anisotropic profiles and smooth surface morphology can be obtained by adjusting the etch conditions. 


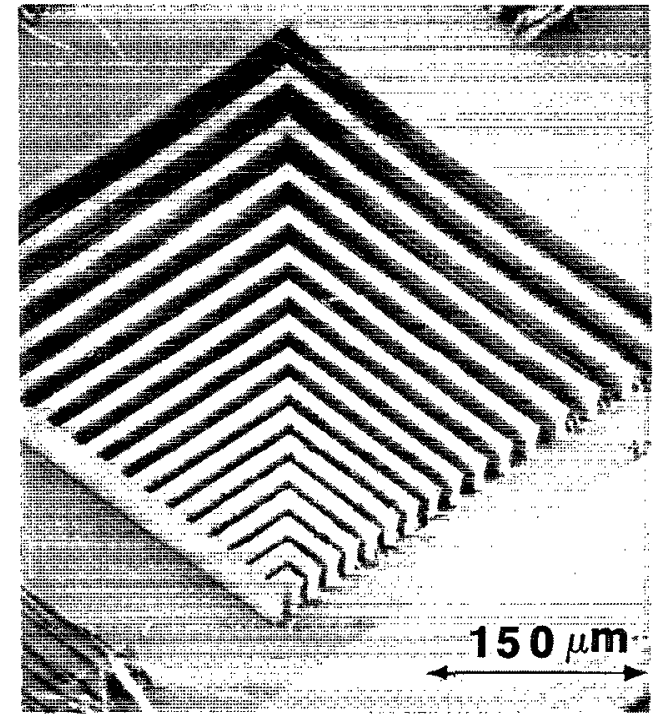

FIG. 1. A $30-\mu \mathrm{m}$-deep polyimide test pattern, produced by etching in an oxygen plasma, for use as a micromold for ceramics.

Micromolding was done using the patterned polyimide wafer as a microscopic "cookie cutter." The surface of the dried tape was softened with a spray of MEK, solvent, and the polyimide wafer was pressed onto the surface with a pressure of $20-30 \mathrm{MPa}$ in a hydraulic press with a dwell time of about $30 \mathrm{~s}$. After drying, the polyimide wafer was peeled from the green tape, leaving an impression of the polyimide pattern on the ceramic tape. The patterned green tape was slowly heated to $600^{\circ} \mathrm{C}$ to remove the EMA binder, then sintered at $1500^{\circ} \mathrm{C}$ for $60 \mathrm{~min}$ The sintered ceria-zirconia was fully dense, with a grain size of $2-4 \mu \mathrm{m}$. Densification of the ceramic resulted in about $20 \%$ linear shrinkage in the plane of the tape.

Figure 1 shows the test pattern, plasma etched $30 \mu \mathrm{m}$ deep in the polyimide. The pattern has 17 lines, each $9 \mu \mathrm{m}$ wide, separated by etched trenches varying from 4 to 25 $\mu \mathrm{m}$ wide. Notice the smooth surfaces of the lines and the area surrounding the pattern. The $0.2 \mu \mathrm{m}$ Ti mask held up well during etching of the polyimide. The etch profile is nearly vertical. The bottoms of the trenches are rather jagged for this pattern, but could be improved by reducing the divergence of the reactive species in the plasma. With lower divergence, the reactive species are more directional and they are closer to normal incidence at the polyimide film. The reduced scattering of the reactive species results in more vertical profile and smoother edges along the trenches. By optimizing the etch conditions such as using lower pressure or higher if power, the directionality of the reactive species can be increased.

Figure 2 is the ceramic replica of the first nine trenches, which resulted in ceramic lines, varying from 7.5 to $22 \mu \mathrm{m}$ wide, separated by $7 \mu \mathrm{m}$ spaces. Note that this is a negative of the polyimide pattern, so the spaces were formed by the polyimide lines and the ceramic lines were made by the polyimide trenchcs. The fired ceramic lines are about $25 \mu \mathrm{m}$ high, so the aspect ratio varied from about 1 to 3 . The features on the ceramic are smaller than the

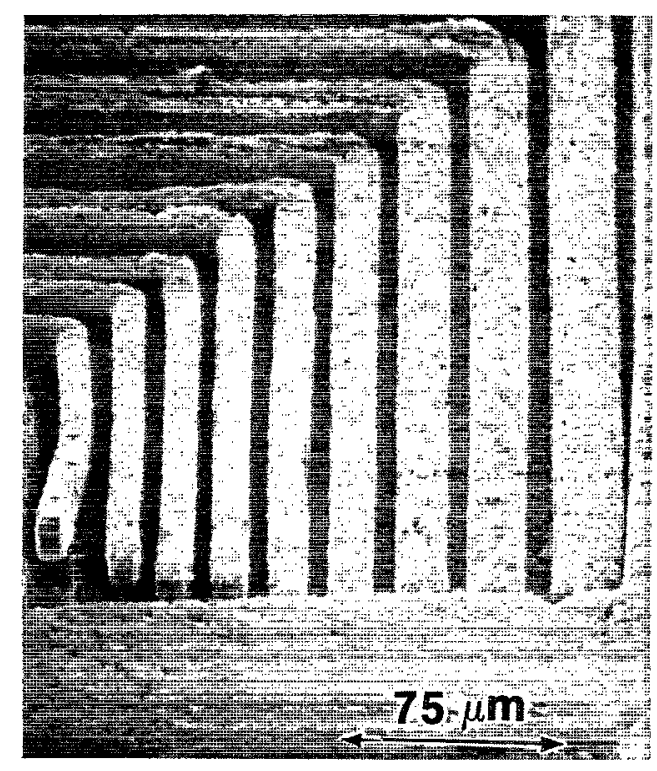

FIG. 2. Ceria-zirconia ceramic replica of the polyimide test pattern, after sintering at $1500^{\circ} \mathrm{C}$.

polyimide pattern, due to the $22 \%$ sintering shrinkage. The spaces between the ceramic lines have smooth bottoms, reflecting the smooth surfaces of the polyimide lines. The ceramic lines have grooves and rough features, replicating the jagged topography of the plasma etched polyimide trenches.

The smaller features of this polyimide test pattern were not successfully replicated in ceramic. The finest intact feature was a $9-\mu \mathrm{m}$-wide ceramic line. The next line, a $7.5-\mu \mathrm{m}$ feature, was replicated, but partially lifted from the ceramic pattern, as shown by the ninth line in Fig. 2 . Ceramic lines smaller than this often pulled off the ceramic tape and remained in the finer trenches of the polyimide pattern.

Patterns with isolated cavities are easier to replicate in ceramic. Figure 3 shows a replica from a different polyimide pattern, with an isolated square-walled cavity having much smoother surfaces. Smooth surfaces were replicated very well in ceria-zirconia, where the surface texture reflects the $2-\mu \mathrm{m}$ grain size of the sintered ceramic. The molded feature in Fig. 3 is a $15-\mu \mathrm{m}$-tall, $26-\mu \mathrm{m}$-wide, and $22-\mu \mathrm{m}$-deep projection from the $15-\mu \mathrm{m}$-tall wall visible in the background. Note the vertical walls, flat top of the feature, and flat bottom of the pattern. The surface texture of the replica is determined by the $2-4 \mu \mathrm{m}$ grain size of the ceria-zirconia ceramic.

The finest feature fabricated to date is illustrated by Fig. 4, a $9-\mu \mathrm{m}$-tall wall about $4 \mu \mathrm{m}$ wide. The top of the wall, at $4 \mu \mathrm{m}$, must have been only 10-12 particle diameters wide in the green state. After sintering and grain growth, it is typically $2-3$ grains wide, with several regions only a single grain wide. Figure 4 also shows rather accurate replication of the horizontal floor (corresponding to the top of the polyimide resist layer), and the vertical 


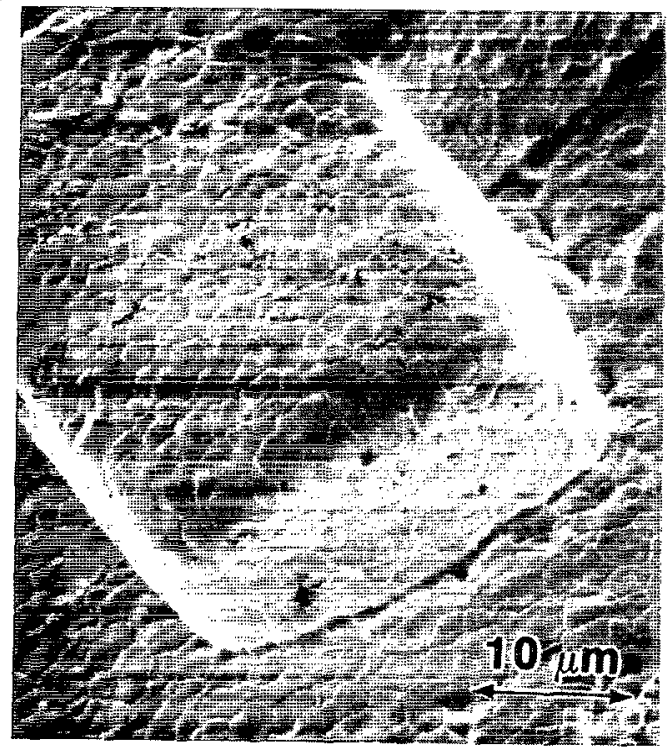

FIG. 3. Micromolded ceria-zirconia feature from another polyimide test pattern, after sintering at $1500^{\circ} \mathrm{C}$.

walls. Note that the radius of curvature of the corner is finer that the sintered grain diameter.

In conclusion, we have fabricated ceria-zirconia ceramics by making simple surface impressions onto soft ceramic green tapes using a plasma-etched polyimide pattern as a micromold. Closely spaced arrays of $25-\mu \mathrm{m}$-high lines could be fabricated having widths as fine as $9 \mu \mathrm{m}$. Smaller lines adhered to the polyimide mold. Features as fine as 4 $\mu \mathrm{m}$ can be made as isolated lines. This method should be applicable for micromolding any ceramic material available as a sufficiently fine powder.

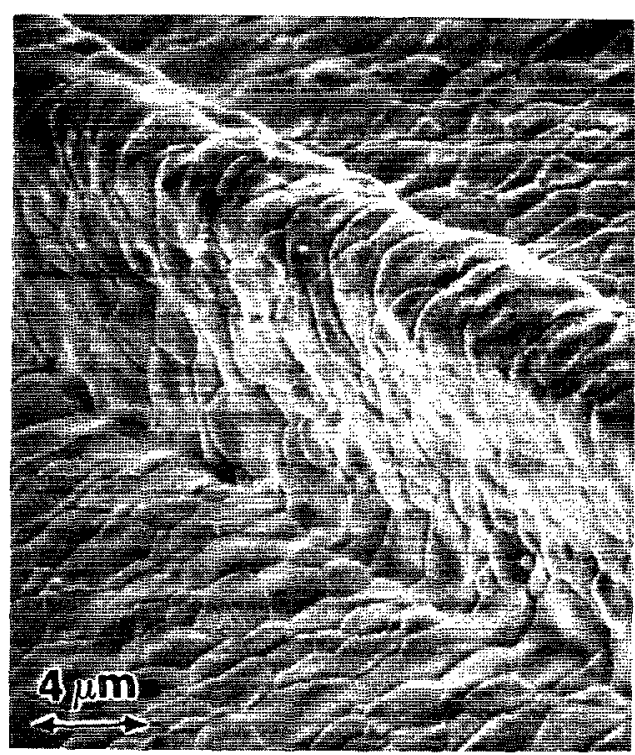

FIG. 4. Isolated wall of ceria-zirconia, $4 \mu \mathrm{m}$ wide by $9 \mu \mathrm{m}$ tall, micromolded from a polyimide pattern.

W.H.J. and S.W.P. would like to acknowledge the support provided by the Advanced Research Project Agency under Contract No. J-FBI-92-149. Partial support (M.O'D.) supplied by the National Institutes of Health under Grant No. CA 54896 is gratefully acknowledged.

${ }^{1}$ M. O'Donnell and L. J. Thomas, IEEE Trans. Ultrason. Ferroelec. Freq. Control 39, 366 (1992).

${ }^{2}$ E. W. Becker, W. Ehrfeld, P. Hagmann, A. Maner, and D. Munchmeyer, Microelectron. Eng. 4, 35 (1986).

${ }^{3}$ A. Furuya, F. Shimokawa, T. Matsuura, and R. Sawada, Proceedings of the Micro-Electro Mechanical Systems Workshop, Ft. Lauderdale, FL, 1993 (IEEE, Piscataway, NJ, 1993), p. 59.

${ }^{4}$ K. T. Sung and S. W. Pang, J. Electrochem. Soc. 139, 3599 (1992). 